\title{
Design of Millimeter-Wave Metamaterials Using Deterministic Full Wave Electromagnetic Simulators and Path of Ascent Statistical Optimizer
}

\author{
D. Staiculescu ${ }^{(1)}$, L. Martin $^{(2)}$, N. Bushyager ${ }^{(1)}$, M. M. Tentzeris ${ }^{(1)}$ \\ (1) Georgia Electronic Design Center, School of ECE, Georgia Tech, Atlanta, GA 30332-0250, U.S.A. \\ (2) Motorola, 8000 Sunrise Blvd., Plantation, FL, 33322, U.S.A.
}

\begin{abstract}
The paper presents the first successful combined use of the Design of Experiments (DOE) and Path of Ascent (POA) approaches in the optimization study for a $40 \mathrm{GHz}$ metamaterial structure implemented in Low Temperature Cofired Ceramic (LTCC) technology. The two figures of merit chosen are the resonating frequency $f_{\text {res }}$ and the corresponding insertion loss $I L$ with the optimization goals of $f_{\text {res }}=40 \mathrm{GHz}$ and minimum $I L$. The electromagnetic performance of the structure is determined with a full wave Transmission Line Matrix (TLM) commercial simulator. The results of these simulations are incorporated into DOE and POA techniques. Statistical models are developed for the two output variables and then applied to optimize the filter.
\end{abstract}

\section{Introduction}

The concept of metamaterials, or left-handed (LH), double negative (DNG), negative refractive index (NRI) materials, has been around since the late 1960's [1], but only recently it has gained a lot of interest since practical implementation solutions emerged [2], [3]. These materials exhibit phase and group velocities of opposite signs and a negative refractive index in certain frequency ranges, both characteristics making them desirable for RF and microwave circuits. Possible applications are phase and dispersion compensation, filters, resonators, couplers and leaky-wave antennas, all applicable in RF integrated modules. One of the implementation approaches starts from the equivalent transmission line model and loads a host line with a dual periodical structure of series capacitors and shunt inductors [4]. The length of the period and the value of the capacitors and inductors determine the frequency band in which the material has LH behavior. One of the main challenges for the high frequency implementation, where the dimensions become smaller and the process design rules become very restrictive, is the choice of the inductor and capacitor geometry to obtain the required left-handed passband and minimum insertion loss at the desired operating frequency. The goal of this paper is to design and optimize, for the first time, a metamaterial structure for a frequency as high as 40 GHz. The current design methods do not take into account the specific effect of each of the factors involved in the design process, the degree these factors interact with each other, and the direction to change these factors for performance optimization. The use of POA (Path of Ascent) optimization, which utilizes DOE (Design of Experiments) and RSM (Response Surface Modeling) and is presented in this paper, enables all these goals to be achieved. The presented methodology integrates simulation, measurements, and statistical tools and is applied for the optimization of a 40
$\mathrm{GHz}$ metamaterial structure on LTCC (Low Temperature Cofired Ceramic).

The hybrid design procedure begins with identifying the parameters and the ranges of variation. The ranges, which define the design space, have to be chosen to include the fabrication tolerances, while providing sufficient variation in performance. The optimization variables are the resonating frequency and the corresponding insertion loss. The results of the simulations are incorporated in DOE and POA [5] statistical topologies. The DOE data is analyzed and then POA is applied to determine a path, which extends beyond the DOE design space, for further optimization of the figures of merit. Using this path, another design space is defined and the process repeats with another full factorial DOE with center points. The process is complete when the performance goal or optimum performance is achieved.

The proposed technique can be applied to any type of designs, especially in complex RF microsystems and packages, where the number of factors increases and it is extremely difficult to optimize using only electromagnetic simulators. It gives a thorough understanding of the system behavior and integrates geometrical, material, and functional parameters altogether. The presented approach is generic and independent of the choice of the electromagnetic simulator and statistical analysis software.

\section{Benchmarking Structure}

The benchmarking structure analyzed in this paper is presented in Figure 1. It is located on the top of a LTCC substrate and consists of a coplanar waveguide (CPW) transmission line periodically loaded with series capacitors and shunt inductors [4]. The series capacitors are implemented as gaps in the signal line and the shunt inductors as shorts to the ground plane. Four such cells were included in the simulated structure.

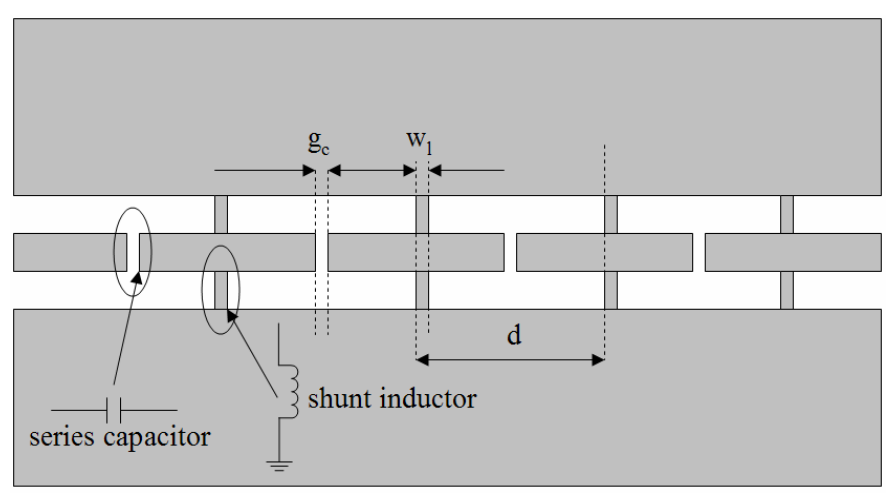

Figure 1. Top view schematic of the metamaterial structure. 
In order to optimize this structure, the range of the parameter values has to be determined. The three design parameters that affect the metamaterial performance are shown in Figure 1: $d$ is the length of the period, $g_{c}$ is the width of the capacitive gap, and $w_{l}$ is the width of the inductive strip. By choosing the parameters in this way, the values of $L$ and $C$ can be controlled by changing respectively only one physical attribute of the circuit, thus the independence of the three input variables is satisfied.

The chosen technology is LTCC, with 5.4 dielectric constant and 0.0015 loss tangent at $35 \mathrm{GHz}$. The substrate is $1100 \mu \mathrm{m}$ thick, which is enough for small variations of thickness to have no significant effect on the field penetration.

First, a preliminary design of the $40 \mathrm{GHz}$ structure has been simulated. Then, the design space for the three parameters has been chosen such that it represents physically realizable values without severely affecting the performance. The ranges for the three input variables are presented in Table 1 . The resonant frequency $f_{\text {res }}$ and the corresponding insertion loss $I L$ are the responses for the statistical models.

Table 1. Ranges for the input variables

\begin{tabular}{|c|c|c|c|}
\hline $\begin{array}{c}\text { Variabl } \\
\mathbf{e}\end{array}$ & $\begin{array}{c}\text { Min } \\
(\boldsymbol{\mu} \mathbf{m})\end{array}$ & $\begin{array}{c}\text { Max } \\
(\boldsymbol{\mu} \mathbf{m})\end{array}$ & $\begin{array}{c}\text { Center Point } \\
(\boldsymbol{\mu} \mathbf{m})\end{array}$ \\
\hline $\boldsymbol{D}$ & 1760 & 1840 & 1800 \\
\hline $\boldsymbol{g}_{\boldsymbol{c}}$ & 130 & 170 & 150 \\
\hline $\boldsymbol{w}_{\boldsymbol{l}}$ & 90 & 110 & 100 \\
\hline
\end{tabular}

The methodology used in the optimization of the metamaterial structure is presented as a flowchart in Figure 2 and illustrated for a simplified experimental design consisting of only two input variables in Figure 3.

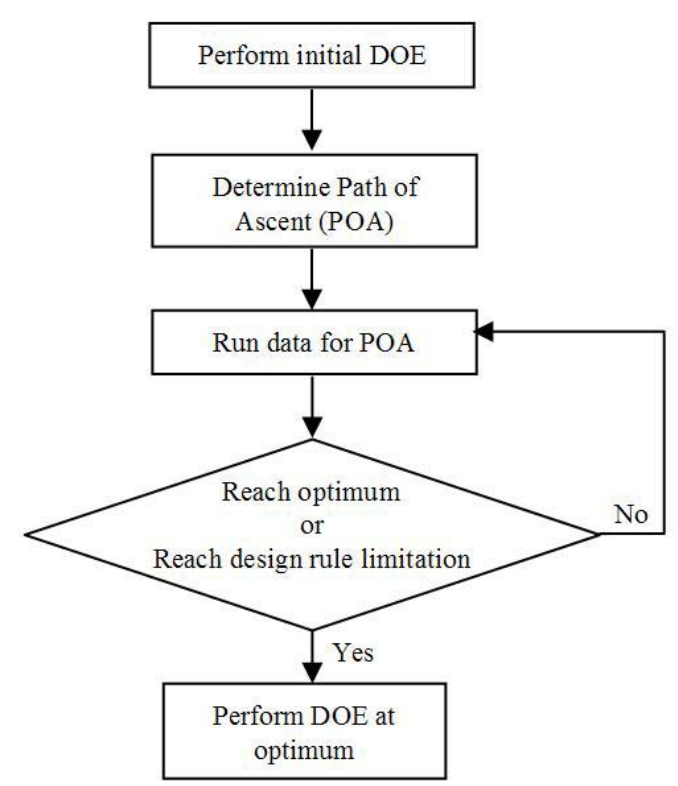

Figure 2. Flowchart for optimization using POA methodology
The experimentation method chosen for the DOE is a full factorial design with center points. The factorial designs are used in experiments involving several factors where the goal is the study of the joint effects of the factors on a response. Prior knowledge of the analyzed system is required for choosing the factors and their studied ranges. The $2^{k}$ factorial design is the simplest one with $k$ factors at 2 levels each. It provides the smallest number of runs for studying $k$ factors and is widely used in factor screening experiments [5].

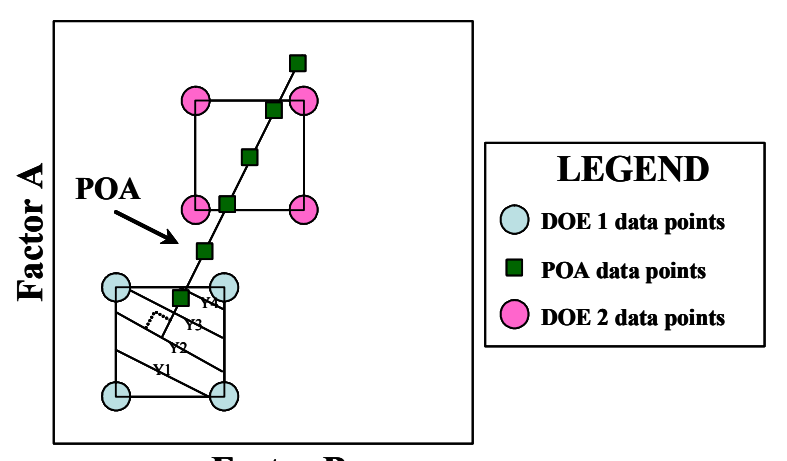

Factor B

Figure 3. Optimization using POA methodology for a simplified experimental design consisting of only two input variables

The design used was a $2^{k}$ factorial design with center points. Center points in the design increase the capability of investigating the model fit, including curvature in the response, and account for variation in the fabrication process of the structure. Since the statistical models are based on deterministic simulations, the variation of the center points were statistically simulated based on a $\pm 5 \mu \mathrm{m}$ tolerance and a $3 \sigma$ fabrication process. Specifically, center points were randomly generated assuming a mean equal to the exact center point value and a standard deviation equal to 1.6667 $\mu \mathrm{m}$.

The statistical analysis of the first order models shows which effects are significant for each of the two figures of merit and the ones that are not significant are eliminated from the final models. POA is then applied to determine a path for further optimization of the figures of merit. Using this path, simulations are run until the optimum or a design rule limitation is reached. The optimum POA point is then used to identify another design space and then another full factorial DOE with center points is performed. The process is complete when the performance goal or optimum performance is achieved.

\section{Statistical model development}

The initial DOE revealed the significant parameters for both figures of merit and then first-order prediction models were developed based on them. At the $95 \%$ confidence level, it was found that all three parameters $d, g_{c}$, and $w_{l}$ were statistically significant for the insertion loss and only $g_{c}$ and $d$ were significant for the resonant frequency $f_{\text {res }}$. Additionally, the model for insertion loss $I L$ was validated for model assumptions of normality and equal variance of the residuals. 
The model for the resonating frequency $f_{\text {res }}$ was validated for normality assumption [5], but there appeared to be curvature due to the center point residuals, when the equal variance assumption was investigated. No lack of fit for either model was confirmed at the $99 \%$ confidence level.

Based on the first-order prediction model for insertion loss $I L$, the POA was determined for optimization of insertion loss, while the predicted resonant frequency was monitored. The fourth step of the POA was found to be optimum, based on decreased insertion loss and constraint of design rules for the input variables.

Based on these POA results, the fourth step became the center point of the second DOE and ranges that were proportional to the first DOE were used for the experimental design. At the 95\% confidence level, it was found that all three parameters $d, g_{c}$, and $w_{l}$ and the interaction of $w_{l}$ and $d$ were statistically significant for insertion loss IL. At the $95 \%$ confidence level, it was found that all three parameters $d, g_{c}$, and $w_{l}$, the interaction of $g_{c}$ and $d$, and the interaction of $w_{l}$ and $d$ were statistically significant for resonant frequency $f_{\text {res }}$. Both models were validated for normality assumption, but there appeared to be a slight funnel of residuals, increasing with increasing response, when the equal variance assumption was investigated. A slight lack of fit for both models was confirmed at the $99 \%$ confidence level. These models are given by the (1)-(2).

$$
\begin{aligned}
& I L=5.88-0.166\left(\frac{d-1875}{40}\right)+0.531\left(\frac{g_{c}-90}{20}\right) \\
& +0.339\left(\frac{w_{l}-81}{10}\right)+0.206\left(\frac{w_{l}-81}{10}\right)\left(\frac{d-1875}{40}\right) \\
& f_{\text {res }}=40.0-0.224\left(\frac{d-1875}{40}\right)+0.216\left(\frac{g_{c}-90}{20}\right) \\
& +0.131\left(\frac{w_{l}-81}{10}\right)+0.234\left(\frac{g_{c}-90}{20}\right)\left(\frac{d-1875}{40}\right) \\
& +0.234\left(\frac{w_{l}-81}{10}\right)\left(\frac{d-1875}{40}\right)
\end{aligned}
$$

Before accepting (1)-(2), the models were confirmed. Confirmations of the models were performed for the following combination of parameters: $d=1896 \mu \mathrm{m}, g_{c}=96$ $\mu \mathrm{m}$, and $w_{l}=84 \mu \mathrm{m}$. This configuration was simulated in the electromagnetic simulator and was also predicted with the developed models. The results of the simulation, compared to the DOE 95\% confidence intervals defined by the lower and upper bounds for the predicted resonant frequency $f_{\text {res }}$ and insertion loss $I L$ are shown in Table 2.
Table 2. Resonant frequency and insertion loss from electromagnetic simulation compared to the DOE $95 \%$ confidence intervals

\begin{tabular}{|c|c|c|}
\hline & $\boldsymbol{f}_{\text {res }}[\mathrm{GHz}]$ & $\boldsymbol{I L}[\mathrm{dB}]$ \\
\hline Simulation & 39.93 & 5.918 \\
\hline DOE lower bound & 39.72 & 5.598 \\
\hline DOE upper bound & 40.43 & 6.582 \\
\hline
\end{tabular}

Because the simulation values fall into the $95 \%$ confidence intervals from the DOE, the DOE models were confirmed and accepted as the final models for optimization.

\section{Model interpretation and optimization}

The models offer the option to optimize performance with respect to either figure of merit or both simultaneously. They can also be used to predict the performance of the system for a specific configuration. In this case, the optimization goals were minimized insertion loss $I L$ for a target resonant frequency of $40 \mathrm{GHz}$. Figure 4 shows the surfaces of possible solutions for the optimization goals. The optimization is done based on the intersection of the surfaces, which is shown in Figure 5. The values that satisfied the optimization conditions within the design space of the second DOE were $d=1915 \mu \mathrm{m}$, $g_{c}=75 \mu \mathrm{m}$, and $w_{l}=75 \mu \mathrm{m}$ leading to the optimized values of the two figures of merit of $I L=4.99$ and $f_{\text {res }}=39.2 \mathrm{GHz}$. Both Figures 3 and 4 show the figures of merit as functions of $g_{c}$ and $w_{l}$ with $d$ fixed at $1915 \mu \mathrm{m}$. Figure 6 shows a plot of the simulated optimized metamaterial structure performance.
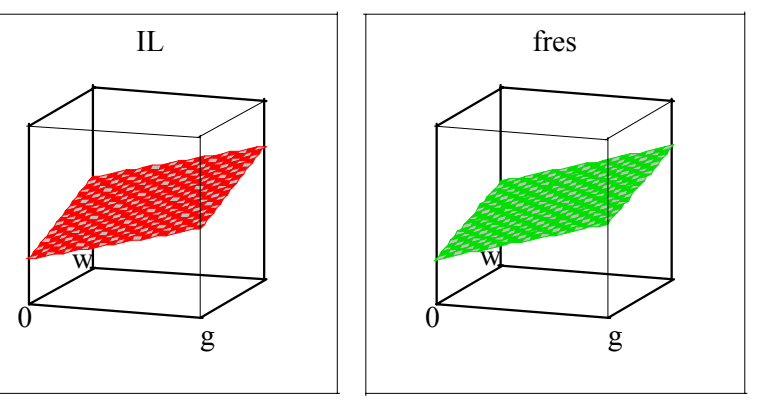

Figure 4. Surfaces representing possible solutions for the optimization goals

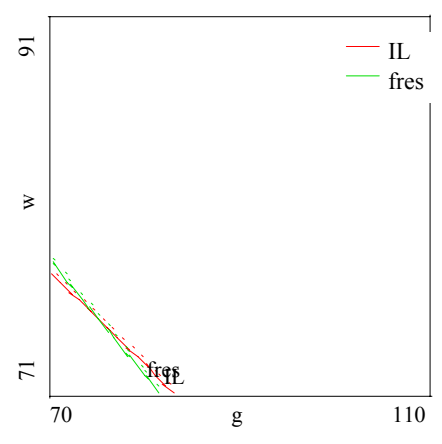

Figure 5. Intersection of the surfaces represents the optimized condition 


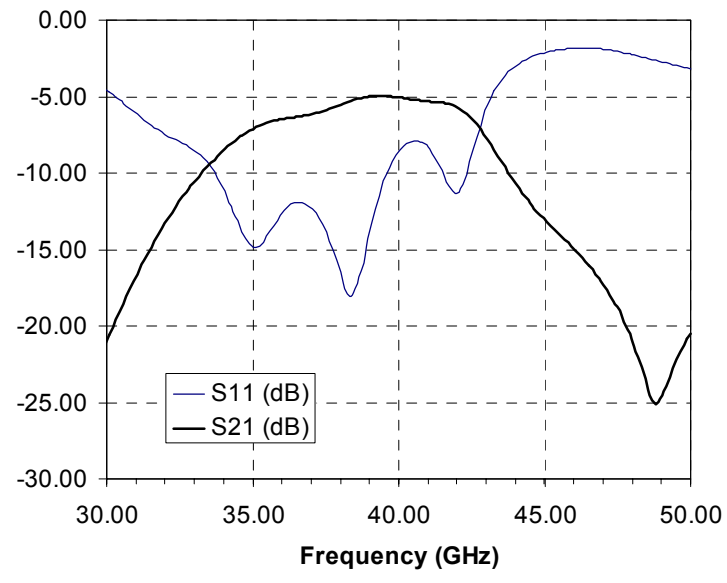

Figure 6. Performance of optimized structure

\section{Conclusions}

This paper presents a method in which deterministic electromagnetic simulation tools and statistical modeling methods can be used to optimize RF components and microsystems. To prove the concept, a benchmarking geometry of a metamaterial structure in LTCC technology was chosen. The two optimized responses were the center frequency and the insertion loss. Three geometric parameters of the structure were chosen as experimental factors. The results of the hybrid electromagnetic-statistical analysis generated statistical models that could be used to predict the filter performance based on the geometry of the structure. These models could then be used to optimize the filter with respect to desired performance, enabling the system-level optimization of the geometry in quick and inexpensive way. In this case, the center frequency was chosen and the insertion loss was minimized to exemplify the possibilities of the method.

The proposed approach can be easily extended to a larger number of design variables and optimized figures of merit. The advantage of the POA approach is that the method provides direction for further optimization, which may extend beyond the initial DOE design space, and is able to distinguish local performance maxima from paths of continued performance optimization. In this way, the behavior of a complex system, such as a 3D multilayer module, could be predicted at the beginning of the design process, leading to a much shorter design cycle of added functions, while achieving design optimization goals in a simple and elegant manner.

\section{Acknowledgments}

The authors wish to acknowledge the support of the NSF CAREER Award ECS-9984761, the NSF Grant ECS0313951, and the GT-Packaging Research Center (PRC).

\section{References}

[1] V. G. Veselago, "The electrodynamics of substances with simultaneously negative values of $\varepsilon$ and $\mu$ ", Sov. Phys. Usp., vol. 10, no.4, pp. 509-514, Jan. - Feb. 1968.

[2] E. Ozbay, K. Ayidin, E. Cubucku, M. Bayindir, "Transmission and Reflection Properties of Composite Double Negative Metamaterials in Free Space", IEEE Trans. Antenna \& Propag., Vol.51, No.10, pp. 25922595, October 2003.

[3] A. Grbic, G. V. Eleftheriades, "Experimental Verification of Backward-Wave Radiation from a Negative refractive Index Material", Journal of App. Phys., Vol.92, No.10, pp.5930-5935, November 2002.

[4] G. V. Eleftheriades, A. K. Iyer, P. C. Kremer, "Planar Negative Refractive Index Media Using Periodically L-C Loaded Transmission Lines", ", IEEE Trans. Microwave Theory \& Tech., Vol.50, No.12, pp.2702-2712, December 2002.

[5] A. Dean et al, Design and Analysis of Experiments, Springer-Verlag New York, Inc., New York, 1999. 